\title{
$\operatorname{con} t-9.20610 \cdots-16$
}

UCRL-JC-111546

PREPRINT

\section{Parametric Instabilities in Large Nonuniform Laser Plasmas.}

\section{H.A. Baldis, D.S. Montgomery, J.D. Moody, C. Labaune \\ S.H. Batha, K.G. Estabrook, R.L. Berger, W.L. Kruer}

This paper was prepared for submittal to the

Proceedings on the 1992 International

Conference on Plasma Physics

Innsbruck, Austria

June 1992

September 1992

This is a preprint of a paper intended for publication in a journal or proceedings. Since changes may be made before publication, this preprint is made available with the understanding that it will not be cited or reproduced without the permission of the author.
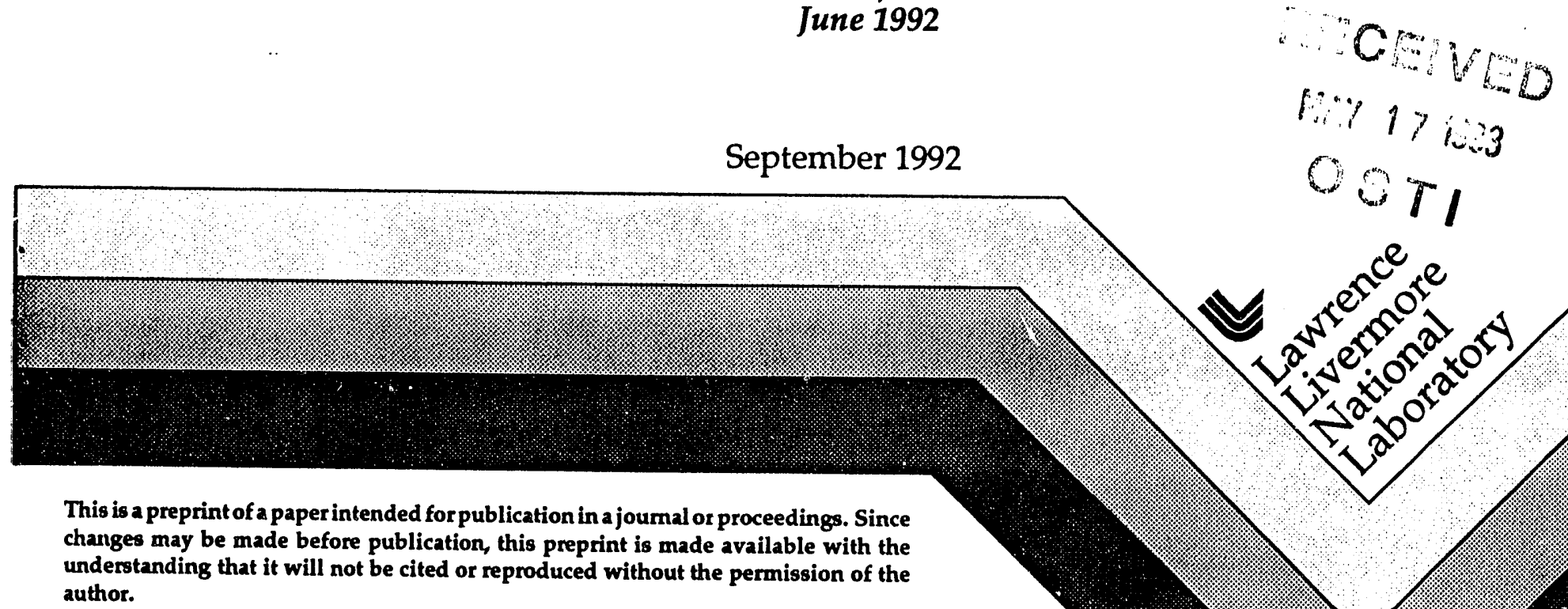
DISCLAIMER

This document was prepared as an account of work sponsored by an agency of the United States Government. Neither the United States Government nor the University of California nor any of their employees, makes any warranty, express or implied, or assumes any legal liability or responsibility for the accuracy, completeness, or usefulness of any information, apparat us, product, or process disclosed, or represents that its use would not infringe privately owned rights. Reference herein to any specific commercial products, process, or service by trade name, trademark, manufacturer, or otherwise, does not necessarily constitute or imply its endorsement, recommendation, or favoring by the United States Government or the University of California. The views and opinions of authors expressed herein do not necessarily state or reflect those of the United States Government or the University of California, and shall not be used for advertising or product endorsement purposes. 
Parametric Instabilities in Large Nonuniform Laser Plasmas

\author{
H.A. Baldis, D.S. Montgomery, J. D. Moody, C. Labaune(a), \\ S.H. Batha ${ }^{(b)}$, K. G. Estabrook, R.L. Berger, W.L. Kruer
}

Lawrence Livermore National Laboratory

P.O. Box 808, Livermore, CA 94550, USA

The study of parametric. instabilities in laser plasmas is of vital importance for inertial confinement fusion (ICF). The long scale-length plasma encountered in the corona of an ICF target provides ideal conditions for the growth of instabilities such as stimulated Brillouin scattering (SBS), stimulated Raman scattering (SRS), and filamentation /1//2/. These instabilities can have detrimental effects in ICF and their characterization and understanding is of importance. Scattering instabilities are driven through a feedback loop by which the beating between the electromagnetic EM fields of the laser and the scattered light matches the frequency of a local longitudinal mode of the plasma. Any process which interferes with the coherence of this mechanism can substantially alter the behavior of the instability.

Of particular interest is the study of laser beam smoothing techniques /3//4/ on parametric instabilities. These techniques are used to improve irradiation uniformity which can suppress hydrodynamic instabilities. Laser beam smoothing techniques have the potential to control the scattering level from parametric instabilities since they provide not only a smoother laser intensity distribution, but also reduced coherence. Beam smoothing techniques that affect the growth of parametric instabilities include spatial smoothing and temporal smoothing by laser bandwidth. Spatial smoothing modifies the phase fronts and temporal distribution of intensities in the focal volume. The transverse intensity spectrum is shifted towards higher spatial wavenumber and can significantly limit the growth of filamentation. Temporal smoothing reduces the coherence time and consequently limits the growth time. Laser bandwidth is required for most smoothing techniques, and can have an independent effect on the instabilities as well. Recent

(a) Ecole Polytechnique, Palaiseau, France.

(b) Plasma Phvsics Research Institute. Universitv of Califomia. USA. 
theoretical work $/ 5 / / 6 /$ has studied the effect of temporal and spatial smoothing on the growth of SRS and SBS.

Two smoothing schemes are presently used: (1) spatial smoothing, which breaks the beam up into fine-scale structures and (2) temporal smoothing, which rapidly varies the fine-scale structure with time. Spatial smoothing was first proposed in 1984 by Kato and co-workers $7 /$ at Osaka University, using a random phase plate (RPP) to break up the beam. Temporal and spatial smoothing was first introduced by the technique called induced spatial incoherence (ISI). This was conceived by Lehmberg and Obenschain /8/ at the Naval Research Laboratory (NRL) in 1983 and was implemented at NRL in 1985. A second method employing bandwidth and RPP, called smoothing by spectral dispersion (SSD), was demonstrated at the University of Rochester's Laboratory for Laser Energetics (LLE) in 1989 by Spupsky and co:-workers /9/.

The first studies of beam smoothing effects on parametric instabilities were done at NRL using the ISI technique. The levels of backscattered SBS and SRS were reduced in plasma produced by spatially and temporally incoherent laser light /10//11/. Strong reduction of the reflectivity of SBS and SRS was also observed in experiments using only RPP to provide spatial smoothing /12//13/. Experiments at Ecole Polytechnique using RPP showed strong reduction for both SBS and SRS $/ 12 /$. For laser intensities $>10^{14} \mathrm{~W} \mathrm{~cm}^{-2}$ a reduction of approximately two orders of magnitude was observed for both instabilities. Diagnostics designed to look at filamentation also indicated a reduction when the RPP was used /14//15/. In a similar experiment at Rutherford /13/ the observed reductions on SBS and SRS were attributed exclusively to filamentation.

Recent experiments at Livermore are designed to characterize the effect of beam smoothing on SBS and SRS. Beam smoothing on Nova consists of a combination of random phase plates (RPP) and smoothing by spectral dispersion (SSD). The experiments use the two beam chamber facility; one beam preforms a plasma, and the second is an interaction beam. This arrangement allows for independent control of the plasma conditions and of the interaction beam. The targets are $\mathrm{Ti}$ or $\mathrm{CH}$ foils. The plasma parameters at the time of arrival of the interaction beam are chosen so as to assure growth of SBE and SRS. The electron density is about $10-15 \%$ critical density, and the electron temperature is about $2 \mathrm{keV}$. The plasma size is of the order of $1 \mathrm{~mm}$. A random phase plate is always used on the plasma forming laser beam to provide uniform illumiration. 
Backscattered light collected by the Nova focusing optics is directed to the diagnostic station by a full aperture beam-splitter and focusing optics. This station contains a high dispersion spectrometer coupled to a streak camera, providing temporally resolved SBS spectra ( $\Delta \lambda \approx 3 \AA, \Delta \tau \approx 0.08 \mathrm{~ns})$. A similar set up, but with a lower dispersion, is used to record the SRS spectra. Photodiodes and calorimeters measure absolute levels of reflectivity. An array of photodiodes at different locations in the chamber monitors the angular distribution of the scattered light. A RPP controls spatial coherence in the interaction beam with hexagonal cells of $7 \mathrm{~mm}$, producing an average intensity on the focal spot of approximately $4 \times 10^{15} \mathrm{~W} / \mathrm{cm}^{2}$.

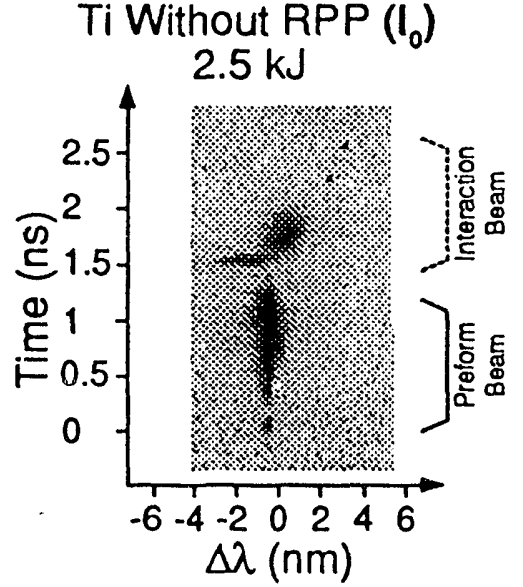

(a)

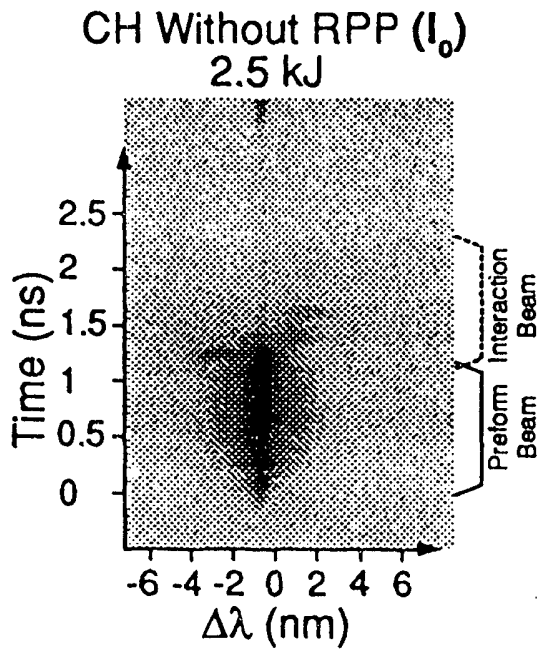

(c)

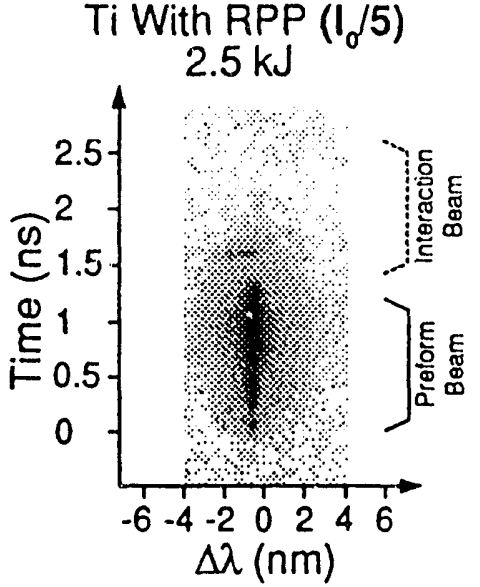

(b)
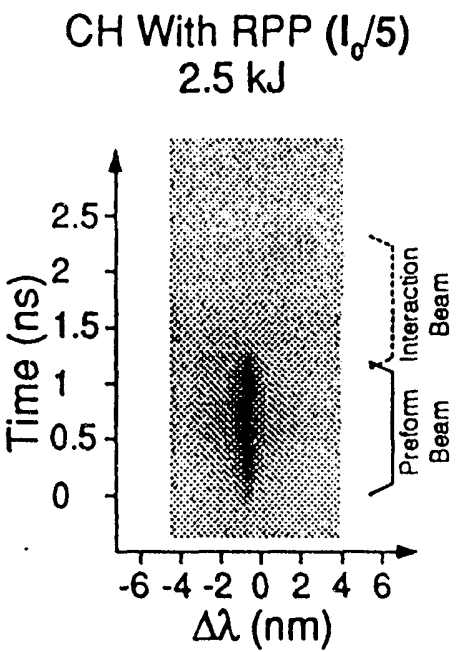

(d)

Figure 1 Time resolved spectra of the preform and backscattered light for (a) Ti target without RPP, (b) Ti target with RPP, (c) CH target without RPP, and (d) CH target with RPP. 
Figure 1 shows the time resolved spectra of SBS backscattered light from a Ti and $\mathrm{CH}$ foil without RPP smoothing [Fig. 1 (a) and (c)] and with RPP smoothing [Fig. 1 (b) and (d)]. The figures show the transmitted light from the preforming pulse that begins at time zero and lasts for approximately $1 \mathrm{~ns}$. The interaction pulse turns on at about 1.4 $\mathrm{ns}$ for $\mathrm{Ti}$ and $1.1 \mathrm{~ns}$ for $\mathrm{CH}$ and lasts for about $1 \mathrm{~ns}$. There is a spectrally broad blue shifted flash at the beginning of the interaction beam turn on which lasts about 100 picoseconds. This flash is followed by a 300 picosecond long feature with a red shift between zero and about $20 \AA$. This feature appears only in cases without RPP smoothing. The average intensity of a smoothed beam is about a factor of five less than an unsmoothed beam. We emphasize this point in the figures by writing the total energy in the interaction beam and the average intensity in the interaction laser spot at the top of each figure. We have made a very recent effort to compare smoothed and unsmoothed cases with the same average intensity by reducing the total energy in the unsmoothed interaction beam (to be published).

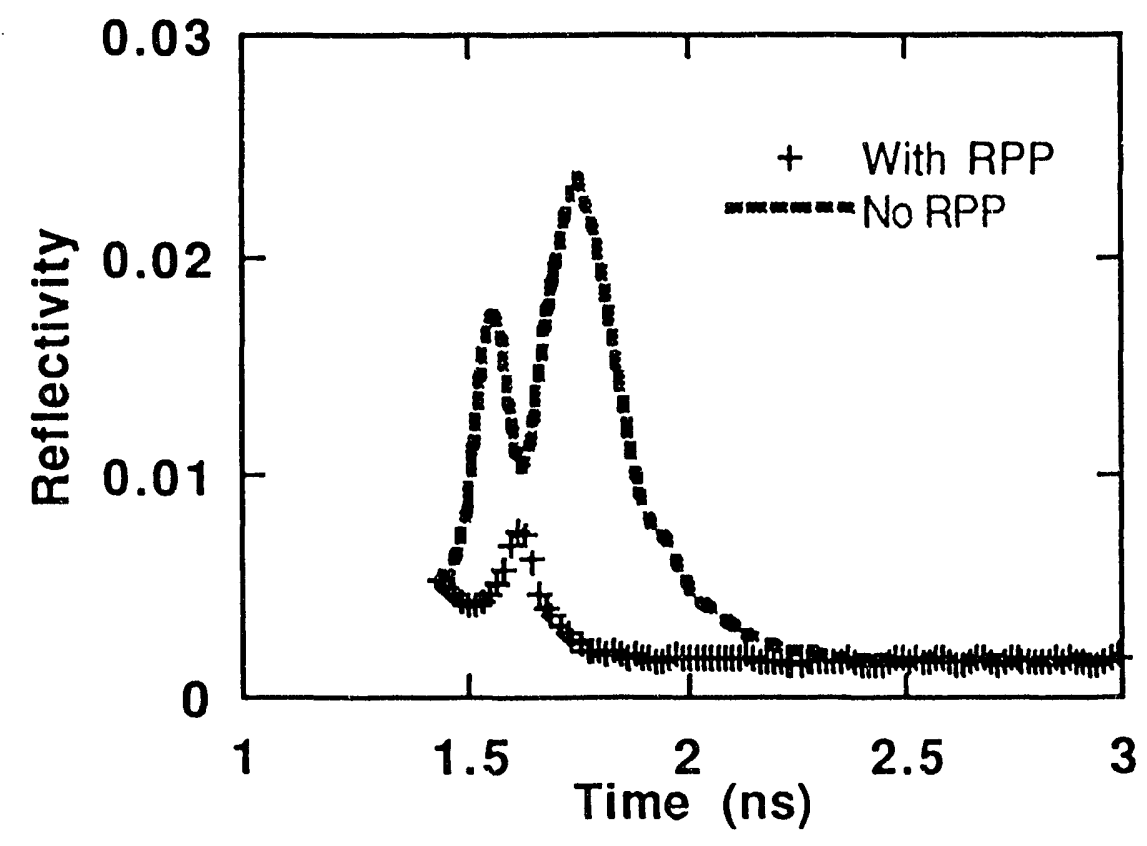

Figure 2 Instantaneous SBS reflectivity for a Ti plasma.

The effectiveness of a RPP in reducing the level of scattering instabilities is determined by analyzing the data shown in Fig. 1 to obtain the time averaged plasma reflectivity. What we mean by the time averaged reflectivity is the ratio of the total energy in the backscattered signal to the total incident interaction beam energy. The backscattered 
light is separated into two components. The first component is the fast feature observed with or without beam smoothing. The second component is the diffuse feature observed only without beam smoothing which exhibits the red shift characteristic of SBS backscattered light from stationary plasmas.

The average reflectivity indicates that beam smoothing using RPP reduces the SBS backscattered light (second component) by at least a factor of 16 to 17 in $\mathrm{Ti}(0.5 \%$ to $0.03 \%$ reflectivity reduction) and $\mathrm{CH}(0.24 \%$ to $0.015 \%$ reflectivity reduction) plasmas. The actual level of reduction may be greater than this since we cannot detect higher levels of reduction with the present experimental set up. This level of reduction is in agreement with previous experimental results reported by Jalinaud /12/, Labaune /15/ and Willi /13/ using smaller scale plasmas. Figure 2 shows the instantaneous SBS reflectivity, with and without RPP for a Ti plasma, obtained from Figure 1. Since the SBS emission has a much shorter duration than the interaction beam ( $1 \mathrm{~ns}$ ), the peak instantaneous reflectivity is larger by at leas a factor of 3 . Preliminary results of recent experiments on Nova show that when higher electron densities are present in the plasma, $\mathrm{n}_{\mathrm{e}}>\mathrm{n}_{\mathrm{cr}} / 4, \mathrm{RPP}$ smoothing has only a minor effect. RPP beam smoothing only causes about a factor of 2 decrease in the first component for both $\mathrm{Ti}(0.2 \%$ to $0.1 \%$ reflectivity reduction) and $\mathrm{CH}(0.16 \%$ to $0.08 \%$ reflectivity reduction) plasmas. Recent experiments also indicate that this first component is only observed when the maximum plasma density is below $n_{c r} / 4$ and when the interaction beam turns on after the preform beam has shut off.

\section{ACKNOWLEDGMENT}

Work performed under the auspices of the U. S. Department of Energy by Lawrence Livermore National Laboratory under contract No. W-7405-Eng-48.

\section{REFERENCES}

/1/ W.L. Kruer, "The Physics of Laser Plasma Interactions"(Addison-Wesley, New York, 1988).

12/ H.A. Baldis, E.M. Campbell, and W.L. Kruer, in Handbook of Plasma Physics, Vol. 3: Physics of Laser Plasma (North Holland, 1991), Chap. 9. 
13/ M. A. Henesian, S. N. Dixit, H. T. Powell, C. E. Thompson, P. J. Wegner and T. L. Weiland, XVIII International Quantum Electronics Conference, 14-19 June 1992, Vienna, Austria, TuE3.

14/ H.T. Powell, S.N. Dixit, and M.A. Henesian, Lawrence Livermore National Laboratory ICF Quarterly Report, 1, 28 (1990) (UCRL-LR-105821-91-1)

15/ D. Pesme, Rapport annuel du GRECO 1987, CNRS-Ecole Polytechnique, France, page 27.

16/ R.L. Berger, Phys. Rev. Lett. 65, 1207 (1990).

n/ Y. Kato, K. Mima, N. Miyanaga, S. Arinaga, Y. Kitagawa, M. Nakatsuka, and C. Yamanaka, Phys. Rev. Lett. 53, 1057, (1984).

18/ R. H. Lehmberg and S. P. Obenschain, Opt. Comm. 46, 27 (1983).

19/ S. Skupsky, R. W. Short, T. Kessler, R. S. Craxton, S. Letzring, and J. M. Soures, J. Appl. Phys. 66, 3456 (1989).

/10/ A.N. Mostovich et al, Phys. Rev. Lett. 59, 1193 (1987).

/11/ S.P. Obenshain et al, Phys. Rev. Lett. 62, 768 (1989).

/12/ T. Jalinaud, S. Baton, C. Labaune, and H.A. Baldis, Rapport Scientifique 1990 du Laboratoire pour L'Utilisation des Lasers Intenses, CNRS-Ecole Polytechnique, France, page 56.

/13/ O. Willi , T. Ashard-rad, S. Coe, A. Giulietti, Phys. Fluids B2, 1318 (1990).

/14/ S. Baton, C. Labaune, T. Jalinaud, H.A. Baldis, 21 ${ }^{\text {rst }}$ European Conference on Laser Interaction with Matter (October 1991), Warsav', Poland.

/15/ C. Labaune, S. D. Baton, T. Jalinaud, H. A. Baldis, D. Pesme, Phys. Fluids B4, 2224, (1992). 

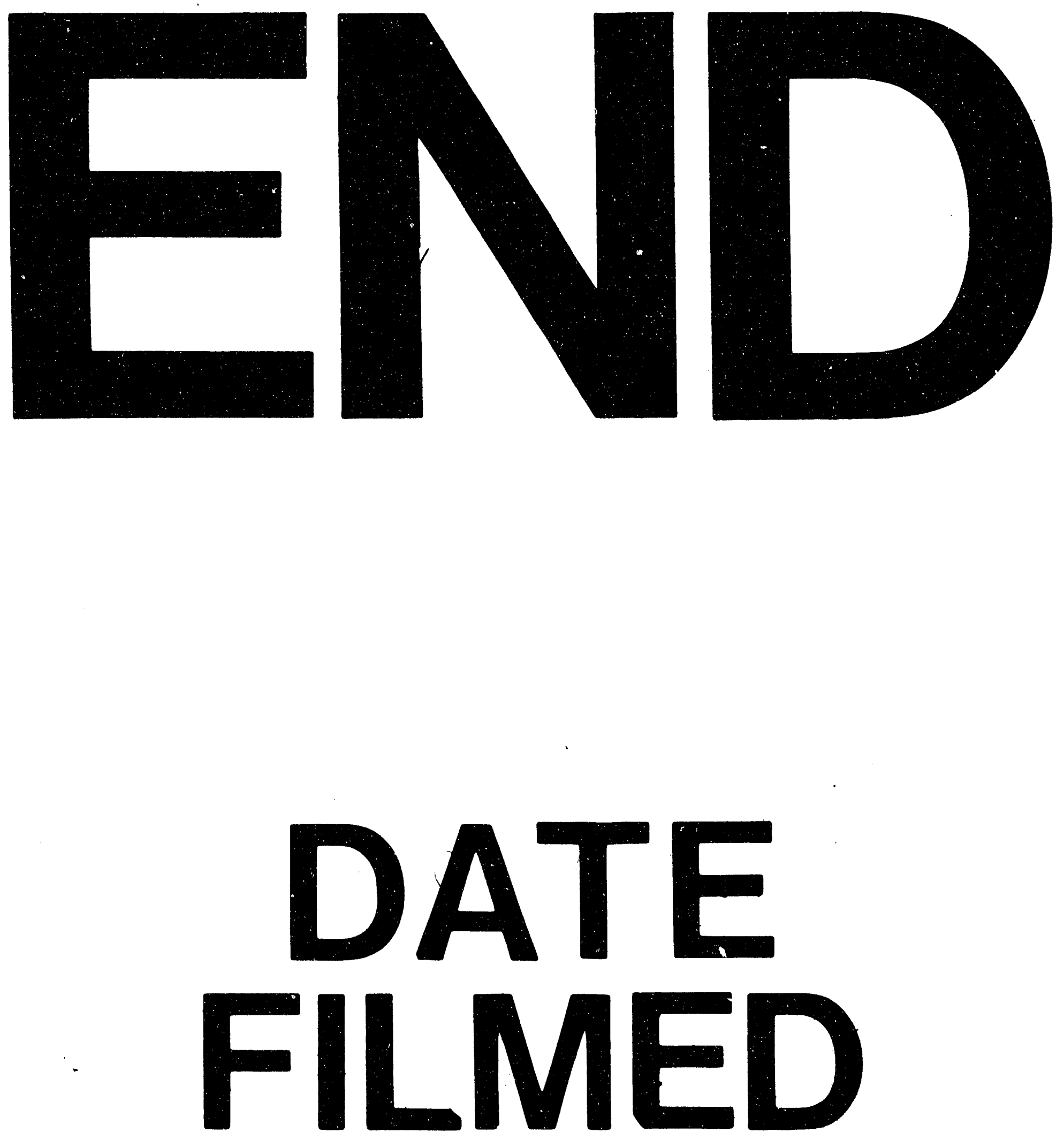

$\uparrow$

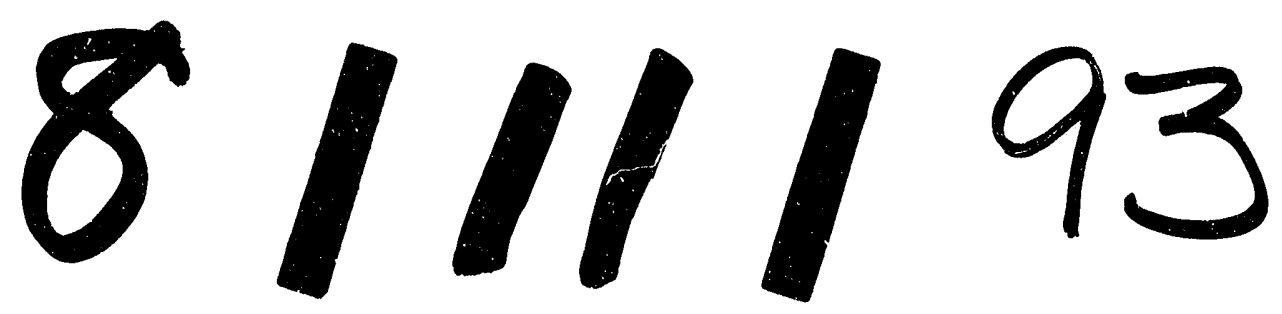


